\title{
DEUX DÉMONSTRATIONS
}

\section{DE LA CONVERGENCE DE CERTAINES FRACTIONS CONTINUES}

\section{PAR}

\section{ANDRE MARKOFF}

a St. PETERSBOURG.

En considérant le développement connu de l'intégrale

en fraction continue

$$
\int_{a}^{b} \frac{f(y)}{z-y} d y
$$

$$
\frac{\mathrm{I}}{\alpha_{1} z+\beta_{1}-\frac{\mathrm{I}}{\alpha_{2} z+\beta_{2}-\frac{\mathrm{I}}{\alpha_{3} z+\beta_{3}-} \cdot}}
$$

supposons les limites $a$ et $b$ réelles de même que toutes les valeurs de la variable d'intégration $y$ et de $\sqrt{f(y)}$.

Nous allons démontrer très simplement, que dans ces suppositions on aura

$$
\int_{a}^{b} \frac{f(y)}{z-y} d y=\lim _{n=\infty} \frac{1}{\alpha_{1} z+\beta_{1}-\frac{1}{\alpha_{2} z+\beta_{2}-}} \cdot \frac{1}{\cdot-\frac{1}{\alpha_{n} z+\beta_{n}}}
$$

pour toutes les valeurs de $z$, qui ne sont pas sur le chemin d'intégration. Acta mathematica. 19. Imprimé le 2 février 1895. 
Rappelons ${ }^{1}$ à ce but, que l'expression

se réduit à la fraction

$$
\frac{1}{\alpha_{1} z+\beta_{1}-\frac{1}{\alpha_{2} z+\beta_{2}-}} \frac{1}{-\frac{1}{\alpha_{n} z+\beta}}
$$

$$
\frac{\varphi_{n}(z)}{\varphi_{n}(z)}
$$

dont le dénominateur $\varphi_{n}(z)$ est une fonction entière de $z$, de degré $n$, satisfaisant aux conditions

$$
\mathrm{o}=\int_{a}^{b} \varphi_{n}(y) f(y) d y=\int_{a}^{b} y \varphi_{n}(y) f(y) d y=\ldots=\int_{a}^{b} y^{n-1} \varphi_{n}(y) f(y) d y,
$$

et le numérateur $\phi_{n}(z)$ se détermine par la formule

$$
\phi_{n}(z)=\int_{a}^{b} \frac{\varphi_{n}(z)-\varphi_{n}(y)}{z-y} f(y) d y
$$

On sait aussi, que l'équation

$$
\varphi_{n}(z)=0
$$

n'a point de racines multiples ou imarginaires et que toutes ses racines

$$
y_{1}, y_{2}, \ldots, y_{n}
$$

sont comprises entre $a$ et $b$.

Il en résulte, que pour chaque fonction entière $\Omega(y)$, de degré moindre que $2 n$, on aura

$$
\int_{a}^{b} Q(y) f(y) d y=\sum \frac{\psi_{n}\left(y_{i}\right)}{\varphi_{n}^{\prime}\left(y_{i}\right)} \Omega\left(y_{i}\right)
$$

en particulier

$$
\int_{a}^{b} f(y) d y=\sum \frac{\phi_{n}\left(y_{i}\right)}{\varphi_{n}^{\prime}\left(y_{i}\right)}
$$

1 C. Possé, Sur quelques applications des fractions continues algébriques, 1886. 
et

$$
\frac{\phi_{n}\left(y_{i}\right)}{\varphi_{n}^{\prime}\left(y_{i}\right)}=\int_{a}^{b}\left\{\frac{\varphi_{n}(y)}{\left(y-y_{i}\right) \varphi_{n}^{\prime}\left(y_{i}\right)}\right\}^{2} f(y) d y>0
$$

D'autre côté l'égalité évidente

nous donne

$$
\frac{\phi_{n}(z)}{\varphi_{n}(z)}=\sum \frac{\phi_{n}\left(y_{i}\right)}{\varphi_{n}^{\prime}\left(y_{i}\right)} \frac{\mathbf{I}}{z-y_{i}}
$$

$$
\int_{a}^{b} \frac{f(y)}{z-y} d y-\frac{\varphi_{n}(z)}{\varphi_{n}(z)}=\int_{a}^{b} \frac{f(y)}{z-y} d y-\sum \frac{\varphi_{n}\left(y_{i}\right)}{\varphi_{n}^{\prime}\left(y_{i}\right)} \frac{\mathbf{I}}{z-y_{i}}
$$

et par conséquent

$$
\begin{aligned}
\int_{a}^{\delta} \frac{f(y)}{z-y} d y-\frac{\phi_{n}(z)}{\varphi_{n}(z)} & =\int_{a}^{b}\left\{\frac{\mathbf{I}}{z-y}-\Omega(y)\right\} f(y) d y \\
& -\sum\left|\frac{\mathbf{I}}{z-y_{i}}-\Omega\left(y_{i}\right)\right| \frac{\phi_{n}\left(y_{i}\right)}{\varphi_{n}^{\prime}\left(y_{i}\right)}
\end{aligned}
$$

quelle que soit la fonction entière $\Omega(y)$ de degré moindre que $2 n$.

Après ces remarques prenons un nombre $x$ quelconque, satisfaisant seulement à l'inégalité

$$
\bmod . \frac{y-x}{z-x}<\mathrm{I}
$$

pour tout le chemin d'intégration, et posons

$$
\Omega(y)=\frac{\mathrm{I}}{z-x}+\frac{y-x}{(z-x)^{2}}+\frac{(y-x)^{2}}{(z-x)^{3}}+\ldots+\frac{(y-x)^{2 n-1}}{(z-x)^{2 n}}
$$

Alors la différence

$$
\frac{\mathrm{I}}{z-y}-g(y)
$$

sera réduite à

$$
\frac{(y-x)^{2 n}}{(z-y)(z-x)^{2 n}}
$$


et par suite on aura

$$
\int_{a}^{b} \frac{f(y)}{z-y} d y-\frac{\varphi_{n}(z)}{\varphi_{n}(z)}=\int_{a}^{b} \frac{(y-x)^{2 n}}{(z-y)(z-x)^{2 n}} f(y) d y-\sum \frac{\left(y_{i}-x\right)^{2 n}}{\left(z-y_{i}\right)(z-x)^{2 n}} \frac{\varphi_{n}\left(y_{i}\right)}{\varphi_{n}^{\prime}\left(y_{i}\right)}
$$

Quant aux expressions

$$
\int_{a}^{b} \frac{(y-x)^{2 n}}{(z-y)(z-x)^{2 n}} f(y) d y \quad \text { et } \quad \sum \frac{\left(y_{i}-x\right)^{2 n}}{\left(z-y_{i}\right)(z-x)^{2 n}} \frac{\varphi_{n}^{\prime}\left(y_{i}\right)}{\varphi_{n}^{\prime}\left(y_{i}\right)}
$$

leurs modules sont plus petits que le produit de l'intégrale

$$
\int_{a}^{b} f(y) d y
$$

par le maximum du module de l'expression

$$
\frac{(y-x)^{2 n}}{(z-y)(z-x)^{2 n}}
$$

sur le chemin de l'intégration.

Or, $x$ étant constant, ce maximum sera si petit qu'on vondra, pour les valeurs de $n$ assez grandes.

Donc la différence

$$
\int_{a}^{b} \frac{f(y)}{z-y} d y-\frac{\varphi_{n}(z)}{\varphi_{n}(z)}
$$

tend vers zéro, à mesure que $n$ croît infiniment. Les considérations précédentes peuvent aussi indiquer une limite supérieure au module de la différence

$$
\int_{a}^{b} \frac{f(y)}{z-y} d y-\frac{\mathbf{1}}{a_{1} z+\beta_{1}-\frac{1}{a_{2} z+\beta_{2}-} \cdot-\frac{1}{a_{n} z+\beta_{n}}}
$$


Deux démonstrations de la convergence de certaines fractions continues.

A cet effet il est important de choisir le nombre $x$ en sorte, que le maximum du module de

soit le plus petit possible.

$$
\frac{y-x}{z-x}
$$

Conformément à cette condition nous posons

$$
x=\frac{a+b}{2},
$$

si $z$ est réel, et

$$
x=\frac{a+b}{2}-d t \sqrt{-1}
$$

si $z$ est un nombre imaginaire:

$$
z=c+d \sqrt{-\mathrm{I}}
$$

en déterminant $t$ comme la racine positive de l'équation

$$
t^{2}+\frac{d^{2}+(c-a)(c-b)}{d^{2}} t-\left(\frac{b-a}{2 d}\right)^{2}=0
$$

Avec la valeur de $x$ choisie par nous, on trouvera que le module de la différence

est. inférieur à

$$
\int_{a}^{b} \frac{f(y)}{z-y} d y-\frac{\mathrm{I}}{\alpha_{1} z+\beta_{1}-\frac{\mathrm{I}}{\alpha_{2} z+\beta_{2}-} \cdot \ddots-\frac{\mathrm{I}}{\alpha_{n} z+\beta_{n}}}
$$

$$
\begin{gathered}
\frac{2}{\sqrt{(c-a)^{2}}+d^{2}}\left(\frac{t}{t+\mathrm{I}}\right)^{n} \int_{a}^{b} f(y) d y \text { ou à } \frac{2}{\sqrt{(c-b)^{2}+d^{2}}}\left(\frac{t}{t+\mathrm{I}}\right)^{n} \int_{a}^{b} f(y) d y \\
\text { ou ’ } \frac{2}{\sqrt{d^{2}}}\left(\frac{t}{t+\mathrm{I}}\right)^{n} \int_{a}^{b} f(y) d y
\end{gathered}
$$

pour

et est inférieur à

$$
z=c+d \sqrt{-1}
$$

$$
\frac{\mathrm{I}}{a-z}\left(\frac{b-a}{2 z-a-b}\right)^{2 n} \int_{a}^{b} f(y) d y \text { ou } \frac{\mathrm{I}}{z-b}\left(\frac{b-a}{2 z-a-b}\right)^{2 n} \int_{a}^{b} f(y) d y
$$


pour les valeurs de $z$ réelles satisfaisant à la condition

$$
(z-a)(z-b)>0 \text {. }
$$

A propos de ces résultats, remarquons que pour les valeurs de $z$ réelles un autre calcul nous donne comme une limite supérieure du même module le produit de

$$
\frac{4(b-a)^{2 n} \int_{a}^{b} f(y) d y}{\{2 z-a-b+2 \sqrt{(z-a)(z-b)}\}^{2 n}+\{2 z-a-b-2 \sqrt{(z-a)(z-b)}\}^{2 n}}
$$

$\operatorname{par} \frac{\mathrm{I}}{a-z}$ ou par $\frac{1}{z-b}$.

La démonstration précédente suppose les limites $a$ et $b$ finies.

Nous allons donner maintenant, pour les valeurs de $z$ réelles, une autre démonstration, laquelle s'étend aux plusieurs cas

$$
\text { de } a=-\infty \text { ou de } b=+\infty \text {. }
$$

Soit pour fixer les idées

$$
a<y_{1}<y_{2}<\ldots<y_{n-1}<y_{n}<b<z .
$$

En posant

$$
\Omega_{0}(y)=\frac{\varphi_{n}^{2}(z)-\varphi_{n}^{2}(y)}{(z-y) \varphi_{n}^{2}(z)}
$$

et

$$
Q_{1}(y)=\frac{\left(y-y_{n}\right) \varphi_{n}^{2}(z)-\left(z-y_{n}\right) \varphi_{n}^{2}(y)}{(z-y)\left(y-y_{n}\right) \varphi_{n}^{2}(z)}
$$

on aura

$$
\begin{gathered}
\int_{a}^{b} \Omega_{0}(y) f(y) d y=\int_{a}^{b} \Omega_{1}(y) f(y) d y=\frac{\psi_{n}(z)}{\varphi_{n}(z)}, \\
\Omega_{0}(y) \leqq \frac{\mathrm{I}}{z-y} \quad \text { pour } a \leqq y \leqq b, \\
Q_{1}(y) \geqq \frac{\mathrm{I}}{z-y} \quad \text { pour } a \leqq y \leqq y_{n}, \\
\Omega_{1}(y) \geqq \frac{\mathrm{I}}{z-y}-\frac{\left(z-y_{n}\right) \varphi_{n}^{2}(b)}{(z-y)\left(b-y_{n}\right) \varphi_{n}^{2}(z)} \quad \text { pour } y_{n}<y \leqq b
\end{gathered}
$$


Deux démonstrations de la convergence de certaines fractions continues.

et par suite

$$
\int_{a}^{b} \frac{f(y)}{z-y} d y>\frac{\varphi_{n}(z)}{\varphi_{n}(z)}>\int_{a}^{b} \frac{f(y)}{z-y} d y-\frac{\left(z-y_{n}\right) \varphi_{n}^{2}(b)}{\left(b-y_{n}\right) \varphi_{n}^{2}(z)} \int_{y_{n}}^{b} \frac{f(y)}{z-y} d y .
$$

La première de ces inégalités suffit pour conclure la convergence de notre fraction continue, eu égard à l'inégalité

Quant à la formule

$$
\frac{\phi_{n+1}(z)}{\varphi_{n+1}(z)}>\frac{\phi_{n}(z)}{\varphi_{n}(z)}
$$

$$
\int_{a}^{b} \frac{f(y)}{z-y} d y=-\frac{\mathbf{I}}{\alpha_{1} z+b_{1}-\frac{\mathbf{I}}{\alpha_{2} z+\beta_{2}-\frac{1}{\alpha_{3} z+\beta_{3}-}}}
$$

elle découle immédiatement de nos inégalités dans tous les cas, où l'on peut démontrer, qu'une (ou toutes les deux) des quantités

$$
\frac{\left(z-y_{n}\right) \varphi_{n}^{2}(b)}{\left(b-y_{n}\right) \varphi_{n}^{2}(z)} \quad \text { et } \quad \int_{y_{n}}^{b} \frac{f(y)}{z-y} d y
$$

est infiniment petite pour $n=\infty$.

Il n'y a pas de difficulté, si $a$ est fini, car

$$
\frac{\left(z-y_{n}\right) \varphi_{n}^{2}(b)}{\left(b-y_{n}\right) \varphi_{n}^{2}(z)}<\left(\frac{b-a}{z-a}\right)^{2 n-1}
$$

En passant au cas de

$$
a=-\infty
$$

nous posons

$$
b=\mathrm{o}, \quad f(y)=e^{y}(-y)^{\lambda} g(y),
$$

$\lambda$ étant constant, et ajoutons les conditions $\lambda+\mathrm{I}>0$ et $g^{\prime}(y)>0$ pour $-\infty<y<0$. Alors, en appliquant à la fonction

$$
V(y, \xi)=e^{y}(-y)^{\lambda}\{g(y)\}^{\xi}
$$


le théorème premier de ma seconde note ${ }^{1}$ Sur les racines de certaines équations, on trouvera, que les racines

$$
y_{1}, y_{2}, \ldots, y_{n}
$$

de l'équation

$$
\varphi_{n}(y)=0
$$

sont plus grandes $(\xi=1)$ que les racines correspondantes $(\xi=0)$

$$
y_{1}^{0}, y_{2}^{0}, \ldots, y_{u}^{0}
$$

de l'équation

$$
e^{-y} y^{-\lambda} \frac{d^{n}}{d y^{n}}\left\{e^{y} y^{\lambda+n}\right\}=0
$$

Par conséquent dans le cas considéré les valeurs de $\int_{y_{n}}^{b} \frac{f(y)}{z-y} d y$ et de $\frac{\left(z-y_{n}\right) \varphi_{n}^{2}(b)}{\left(b-y_{n}\right) \varphi_{n}^{2}(z)}$ sont respectivement inférieures à $\int_{y^{0}}^{b} \frac{f(y)}{z-y} d y$ et à $\left(\frac{-y_{1}^{0}}{z-y_{1}^{0}}\right)^{2} \cdots\left(\frac{-y_{n-1}^{0}}{z-y_{n-1}^{0}}\right)^{2}\left(\frac{-y_{n}^{0}}{z-y_{n}^{0}}\right)$. Or l'expression

$$
\left(\frac{-y_{1}^{0}}{z-y_{1}^{0}}\right)^{2} \cdots\left(\frac{-y_{n-1}^{0}}{z-y_{n-1}^{0}}\right)^{2}\left(\frac{-y_{n}^{0}}{z-y_{n}^{0}}\right)
$$

égale à

$$
\left(\mathrm{I}-\frac{z}{y_{n}^{0}}\right)\left|\mathrm{I}+\frac{n}{\lambda+\mathrm{I}} z+\frac{n(n-1)}{(\lambda+1)(\lambda+2)} \frac{z^{2}}{\mathrm{I} \cdot 2}+\ldots\right|^{-2}
$$

est plus petite que la suivante

$$
\left\{\mathrm{I}+\frac{n}{\lambda+\mathrm{I}} z+\frac{n(n-1)}{(\lambda+1)(\lambda+2)} \frac{z^{2}}{\mathrm{I} \cdot 2}+\frac{n(n-1)(n-2)}{(\lambda+1)(\lambda+2)(\lambda+3)} \frac{z^{3}}{\mathrm{I} \cdot 2 \cdot 3}+\ldots\right\}^{-1}
$$

laquelle devient infiniment petite pour $n=\infty$. Donc l'intégrale

$$
\int_{-\infty}^{0} \frac{e^{y}(-y)^{\lambda} g(y)}{z-y} d y
$$

'Mathematische Annalen, Bd. 27. 
Deux démonstrations de la convergence de certaines fractions continues.

se développe en fraction continue

$$
\frac{1}{\alpha_{1} z+\beta_{1}-\frac{1}{\alpha_{2} z+\beta_{1}-\frac{1}{\alpha_{3} z+\beta_{3}-}}}
$$

convergente pour toutes les valeurs de $z$ réelles et positives, si $g(y)>0$ et si les intégrales

$$
\int_{-\infty}^{0} e^{y}(-y)^{\lambda} g(y) d y, \int_{-\infty}^{0} e^{y}(-y)^{\lambda+1} g(y) d y, \ldots
$$

ont un sens.

Et nous pouvons assurer, que cette fraction continue est égale à l'intégrale considérée au moins dans les cas où l'on a

$$
\lambda+\mathrm{I}>0 \text { et } g^{\prime}(y)>0 \text { pour }-\infty<y \leqq 0 \text {; }
$$

dans ces cas la différence

$$
\int_{-\infty}^{0} \frac{e^{y}(-y)^{\lambda} g(y)}{z-y} d y-\frac{\mathrm{I}}{\alpha_{1} z+\beta_{1}-\frac{\mathrm{I}}{\alpha_{2} z+\beta_{2}-} \cdot \because-\frac{\mathrm{I}}{\alpha_{n} z+\beta_{n}}}
$$

est moindre que

$$
\frac{\left(\mathrm{I}-\frac{z}{y_{n}^{0}}\right) \int_{y_{n}^{0}}^{0} \frac{e^{y}(-y)^{\lambda} g(y)}{z-y} d y}{\left[\mathrm{I}+\frac{n}{\lambda+\mathrm{I}} z+\frac{n(n-\mathrm{I})}{(\lambda+\mathrm{I})(\lambda+2)} \frac{z^{2}}{\mathrm{I} \cdot 2}+\left.\ldots\right|^{2}\right.},
$$

- $y_{n}^{0}$ étant la plus petite racine de l'équation

$$
\mathrm{I}-\frac{n}{\lambda+1} z+\frac{n(n-1)}{(\lambda+1)(\lambda+2)} \frac{z^{2}}{\mathrm{I} \cdot 2}-\ldots=0
$$



simple:

On peut aller plus loin en démontrant ce théorème important et

Théorème. Si deux fonctions réelles

$$
f^{0}(y) \text { et } f(y)
$$

d'une variable réelle $y$ satisfont aux inégalités

$$
f^{0}(y)>f(y)>0
$$

pour toutes les valeurs de $y$, comprises entre $a$ et $b$; en développant les intégrales

$$
\int_{a}^{b} \frac{f^{0}(y)}{z-y} d y \quad \text { et } \quad \int_{a}^{b} \frac{f(y)}{z-y} d y
$$

en les fractions continues

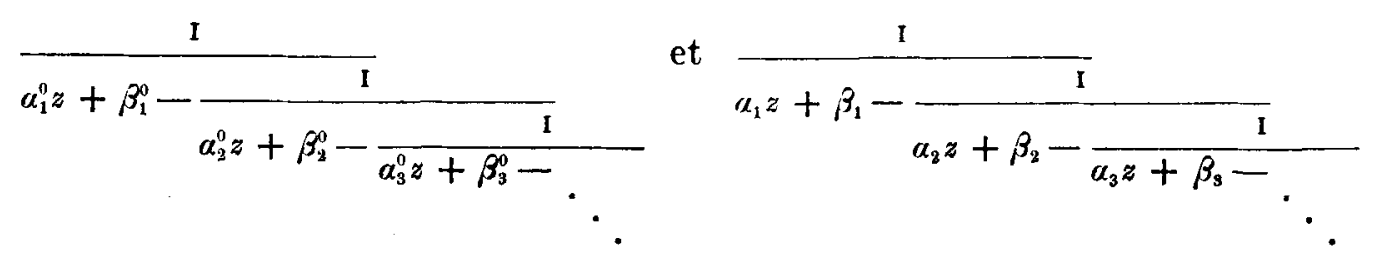

on aura

$$
\int_{a}^{b} \frac{f^{0}(y)}{z-y} d y-\frac{\mathrm{I}}{\alpha_{1}^{0} z+\beta_{1}^{0}-\frac{1}{\alpha_{2}^{0} z+\beta_{2}^{0}-1}} \quad>\int_{a}^{b} \frac{f(y)}{z-y} d y-\frac{\mathrm{I}}{\alpha_{1} z+\beta_{1}-\frac{\mathrm{I}}{\alpha_{2} z+\beta_{2}-} \cdot \frac{\mathrm{I}}{\alpha_{n}^{0} z+\beta_{n}^{0}}}
$$

pour $z>b$ (dans le cas de $z<a$ on doit changer le signe $>$ en $<$ ).

Pour démontrer notre théorème posons

$$
V(y, \xi)=f^{0}(y)+\xi\left[f(y)-f^{0}(y)\right]
$$

et considérons la fraction

$$
\frac{\psi_{n}(z, \xi)}{\varphi_{n}(z, \xi)}
$$


Deux démonstrations de la convergence de certaines fractions continues.

dont le dénominateur $\varphi_{n}(z, \xi)$ est une fonction entière de $z$, de degré $n$, satisfaisant aux équations

$$
\begin{aligned}
\circ=\int_{a}^{b} \varphi_{n}(y, \xi) & V(y, \xi) d y=\int_{a}^{b} y \varphi_{n}(y, \xi) V(y, \xi) d y=\ldots \\
= & \int_{a}^{b} y^{n-1} \varphi_{n}(y, \xi) V(y, \xi) d y
\end{aligned}
$$

et le numérateur $\phi_{n}(z, \xi)$ se détermine par la formule

$$
\phi_{n}(z, \xi)=\int_{a}^{b} \frac{\varphi_{n}(z, \xi)-\varphi_{n}(y, \xi)}{z-y} V(y, \xi) d y .
$$

Cela posé, l'inégalité qu'il faut démontrer, deviendra

$$
\int_{a}^{b} \frac{V(y, 0)}{z-y} d y-\frac{\phi_{n}(z, 0)}{\varphi_{n}(z, 0)}>\int_{a}^{b} \frac{\nabla(y, 1)}{z-y} d y-\frac{\psi_{n}(z, \mathfrak{l})}{\varphi_{n}(z, \mathrm{I})} .
$$

Or on aura

$$
\int_{a}^{b} \frac{V(y, \xi)}{z-y} d y-\frac{\varphi_{n}(z, \xi)}{\varphi_{n}(z, \xi)}=\int_{a}^{b} \frac{\varphi_{n}^{2}(y, \xi)}{\varphi_{n}^{2}(z, \xi)} \frac{V(y, \xi)}{z-y} d y
$$

et

$$
\begin{gathered}
\frac{d}{d \xi} \int_{a}^{b} \frac{\varphi_{n}^{2}(y, \xi)}{\varphi_{n}^{2}(z, \xi)} \frac{\nabla(y, \xi)}{z-y} d y \\
=2 \int_{a}^{b} V(y, \xi) \frac{\varphi_{n}(y, \xi)}{\varphi_{n}^{3}(z, \xi)} \frac{\varphi_{n}(z, \xi) \frac{\partial \varphi_{n}(y, \xi)}{\partial \xi}-\varphi_{n}(y, \xi) \frac{\partial \varphi_{n}(z, \xi)}{\partial \xi}}{z-y} d y \\
+\int_{a}^{b} \frac{\varphi_{n}^{2}(y, \xi)}{\varphi_{n}^{2}(z, \xi)} \frac{\frac{\partial V(y, \xi)}{\partial \xi}}{z-y} d y \\
=\int_{a}^{b} \frac{\varphi_{n}^{2}(y, \xi)}{\varphi_{n}^{2}(z, \xi)} \frac{f(y)-f^{0}(y)}{z-y} d y<0,
\end{gathered}
$$

d'où l'on tirera notre inégalité immédiatement. 
En s'arrêtant au cas, où

$$
\begin{gathered}
a=-\infty, \quad b=0, \quad f^{0}(y)=e^{y}(-y)^{\lambda}, \quad f(y)=e^{y}(-y)^{\lambda} g(y), \\
\lambda+\mathrm{I}>0, z>0 \quad \text { et } \quad \mathrm{o}<g(y)<\mathrm{I} \quad \text { pour }-\infty<y \leqq 0,
\end{gathered}
$$

on trouvera, que la différence entre l'intégrale

$$
\int_{-\infty}^{0} \frac{e^{y}(-y)^{\lambda} g(y)}{z-y} d y
$$

et la réduite

$$
\frac{1}{\alpha_{1} z+\beta_{1}-\frac{1}{\alpha_{2} z+\beta_{2}-}} \frac{1-\frac{1}{\alpha_{n} z+\beta_{n}}}{\alpha_{n}}
$$

de la fraction continue correspondante n'excède pas

$$
\left.\frac{\mathrm{I} \cdot 2 \cdot 3 \ldots n \Gamma(\lambda+1)}{(\lambda+\mathrm{I})(\lambda+2) \ldots(\lambda+n) z} \mid \mathrm{I}+\frac{n}{\lambda+1} z+\frac{n(n-1)}{(\lambda+1)(\lambda+2)} \frac{z^{2}}{1 \cdot 2}+\ldots\right\}^{-2} .
$$

